\title{
Simulating LTE Mobility Management in presence of coverage holes with ns-3
}

\author{
Zoraze Ali, Nicola Baldo, Josep Mangues-Bafalluy, Lorenza Giupponi \\ Mobile Networks Department \\ Centre Tecnològic de Telecomunicacions de Catalunya (CTTC) \\ 08860 Castelldefels, Barcelona, Spain \\ \{zoraze.ali, nicola.baldo, josep.mangues, lorenza.giupponi\}@cttc.cat
}

\begin{abstract}
In this paper, we present our approach to simulate mobility management scenarios for LTE heterogeneous network deployments defined by challenging radio propagation scenarios, such as the presence of coverage holes. We focus on the LTE module of ns-3, which is also known as LENA. Our contribution is twofold. On one hand, we propose a set of new features for the LTE module, including a new model for simulating obstacles blocking the propagation of radio signals, and a handover model suitable for the offline evaluation of mobility management solutions. On the other hand, we describe in detail the setup of the simulation scenario, highlighting the challenges that we faced during the implementation and discussing the chosen configuration parameters. Finally, we present some simulation results that we obtained with the proposed approach.
\end{abstract}

\section{Categories and Subject Descriptors}

1.6.5 [Simulation and Modeling]: Model DevelopmentModeling methodologies; 1.6.7 [Simulation and Modeling]: [Simulation Support-System Environments]

\section{General Terms}

Performance, Design, Experimentation, Verification

\section{Keywords}

LTE, Handover, Mobility, ns-3, Simulator

\section{INTRODUCTION}

In order to face the increasing demand for bandwidth caused by mobile data applications and value added mobile services, $4 \mathrm{G} /$ LTE network deployments are based on the emerging paradigm of heterogeneous networks, which consists in deploying multiple layers of cells of different sizes (macro, micro, small) in order to increase the network capacity.

In contrast with traditional macro cell deployments, where the wide use of above-rooftop-level base stations makes prop- agation relatively well understood and predictable, heterogeneous networks are defined by a highly complex and largely unpredictable propagation environment. For example, outdoor small cell base stations are typically placed below the rooftop level, and hence their coverage is highly affected by the surrounding obstacles, buildings, etc. A cell that is providing coverage at a certain moment might suddenly become unavailable as the user turns around a corner or moves behind an obstacle. Additionally, the low transmission power and short coverage range, combined with such complex propagation effects, exacerbates the uncertainty on whether a given cell could continue providing good coverage for a certain user that is moving, even when the current signal strength is good.

State-of-the-art handover algorithms usually rely on standard User Equipment (UE) measurements, e.g., the A3 event [5], to trigger handover. In traditional macro cell scenarios, it is sufficient to optimize event trigger parameters, e.g., Hysteresis, Time-to-Trigger and Cell individual Offset $[8,9]$, in order to obtain a good performing Mobility Management. However, in the challenging propagation scenario typical of heterogeneous networks, such state-of-the-art handover schemes are not expected to work properly.

In particular, these schemes typically consider handover to the target cell as being successful if neither Radio Resource Control (RRC) connection re-establishment nor Radio Link Failure (RLF) occur upon handover execution, and the event trigger parameter are tuned for this purpose. As a result, in scenarios where handover to the strongest neighbor cell is successful but a while after handover, the same cell get completely blocked by an obstacle, state-of-the-art handover algorithms are likely to lead to a severe degradation of quality of experience, due to the unpredicted cell outage. Because of this, it is of interest to investigate advanced mobility management algorithms that can take a smarter decision in a heterogeneous network deployments considering the experience gained in previous handover events. If users handing over to a certain target cell experience an outage after the handover event, it would be appropriate to select a different target cell that can provide a more sustained coverage in spite of an initially weaker signal upon the decision. With this objective, solutions based on machine learning and big data analytics appear particularly promising [6].

In order to support the design of these advanced LTE mobility management solutions, it is important to be able to 


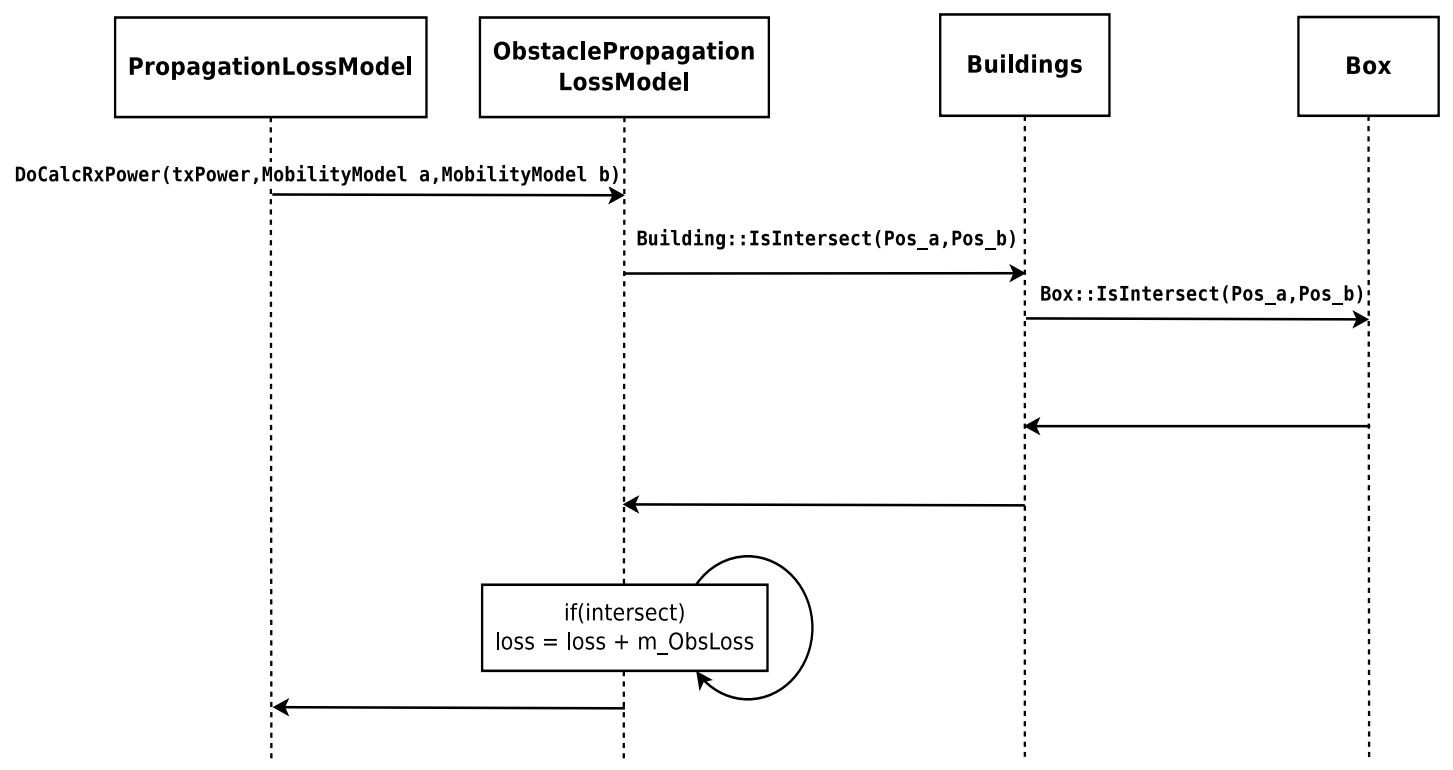

Figure 1: Sequence diagram for calculating obstacle Path Loss

simulate LTE handover scenarios with the complex propagation conditions, in order to better explore the limitations of state-of-the-art approaches and to evaluate the performance of candidate alternative algorithms. We base this study on the LTE module of ns-3, also known as LENA [2], which is a very commonly used LTE network simulation platform that allows simulation of end to end LTE heterogeneous networks. This module is based on an industrial API (the small cell forum LTE MAC Scheduler interface specification) and because of this the protocol stack model is very similar to actual protocol implementations found in commercial products. It also include key aspects such as handover, fractional frequency reuse and support for simulating the buildings in a scenario. However, it is currently not possible to use LENA to simulate coverage holes. Additionally, the preliminary evaluation of a machine learning approach to mobility management would be more easily performed by evaluating offline a large set of different alternative handover decisions; unfortunately, this is not possible with the handover algorithms currently implemented in LENA, which always select the same target base station when facing the same handover conditions. To overcome these limitations, in this paper we propose the following contributions:

1. A model for the simulation of obstacles potentially blocking the propagation of radio signals.

2. A deterministic handover algorithm that can be used for the offline evaluation of learning-based handover algorithms.

3. A simulation scenario for handover in the presence of a coverage hole due to an obstacle.

The source code of this module and simulation is publicly available $\mathrm{at}^{1}$ for use and reproduction of results.

\footnotetext{
${ }^{1}$ https://bitbucket.org/zoraze_cttc/ns-3-dev-obstacle
}

The rest of the paper is organized as follows. Section 2 is dedicated to the contribution $1 \& 2$, together with the implementation of chaining the path loss models. Section $3 \&$ 4 are dedicated to the 3rd contribution, with the configuration of important parts of our simulation and the simulation results, respectively. Finally Section 5 concludes the paper.

\section{NEW DEVELOPED FEATURES}

This section describes the new implemented features in ns-3, for this study.

\subsection{Obstacle Model}

Our approach to implement the obstacle model and to achieve the desired behavior of coverage holes is to use the existing "Buildings" module of ns-3 [1]. The structure of these buildings is defined by a 3D axis aligned box defined by the "Box" class. By doing so, we take advantage of their current functionalities, e.g. maintaining the list of all the obstacles in a simulation, assigning the unique id to an obstacle and using the function "IsInside", provided by the Box class, which indicates whether a node is inside the box or not.

The coverage holes are simulated as follows: if the line segment between the two nodes intersects the box, or any of the nodes is located inside the box, then the transmission is attenuated by adding a significant propagation path loss value. To implement this approach we need to check whether the signal between the two nodes is blocked by building or not but ns-3 does not provide any function for this. Therefore, we extend the functionality of the "Box" class to check the intersection of a line segment between two nodes and the box, based on [7], considering a 3D box.

The obstacle path loss model implemented in this paper is inherited from "propagation-loss-model" class, in intention to chain our path loss model with existing path loss models as explained in the next subsection. The new path loss 
model iterates through the list of all the obstacles created during the simulation, to check for an intersection between the line segment and the box. Upon the intersection it adds the path loss and returns the received power as shown in Fig. 1.

\subsection{Chaining Propagation Loss Models}

The obstacle propagation model described here is a single frequency path loss model. Therefore, we need to chain our model with other path loss models to avoid the flat frequency response. Following is the method to enable the chaining of path loss models in LTE module of ns-3:

- In LTE module the configuration of the path loss model is done by the LteHelper. Besides the conventional way of setting the path loss model through this helper class, we add the functionality for chaining two path loss models. We implement a function AddPropagationLoss, which takes the path loss model and its attribute i.e., a loss value from the user, and push it into a vector. At the time when LteHelper::DoInitialize is called, it checks the status of this vector and upon finding a path loss model to chain, it simply calls the SetNext function of PropagationLossModel class to chain two desired models.

\subsection{Deterministic Handover Algorithm}

In this paper, we have implemented the A2 event triggered deterministic handover algorithm. The purpose of this deterministic handover algorithm is to allow the offline evaluation of the performance of different handover algorithms for each possible target eNBs. The important point while implementing any handover algorithm in ns-3 is the configuration of the UE measurement report for the handover. Our handover algorithm is based on the Reference Signal Received Power (RSRP), which is configurable by setting the triggerQuantity attribute of the UE measurement report. The RSRP threshold value, for which the A2 event is triggered, is also configured through this report. When the algorithm receives the A2 event based measurement report from the UE, it calls the function EvaluateHandover. This function first checks the availability of neighbor cells for handover. If it finds any neighbor and its information e.g the cell id and RSRP, it checks if the TargetCellId is assigned by the user or not. If it is, the BestNeighborCellId is set equal to the user defined target cell id, and the handover is triggered. In case, if the target cell id is not defined by the user it triggers the handover in nondeterministic way, toward the strongest neighbor as shown in Fig. 3.

\section{SIMULATION CONFIGURATION}

This section provides the details to configure the important parts of our simulation to achieve the desired scenario topology. Table. 1 shows the simulation parameters.

\subsection{Scenario Topology}

The scenario topology consists of three macro eNBs, 3 UEs and a coverage hole in the coverage area of eNB2, as shown in the Radio Environment Map in Fig. 2. Each eNB is serving one UE performing TCP file download from the remote host, where UE1(attached to eNB1) is moving around, and other 2 UEs are stationary. The mobility of UE1 is defined in such a way that it picks a fixed starting point close to eNB1 and a random angle to move away from the source eNB following the straight line. This simulation scenario also uses the "Hard fractional frequency reuse" feature of ns-3 to overcome the interference caused by neighboring cells. We note that, the actual deployments are also expected to adopt the similar approach to avoid performance degradation at cell edges. The simulation is consists of 200 runs of a deterministic handover. Each run is repeated twice, first targeting eNB2 and then eNB3 to measure a quality of experience metric, which we selected to be a file download time. The rationale behind selecting TCP file download is that, TCP is the widely used transport protocol for many interactive internet applications, e.g., Telnet and the Web. Secondly, investigating the QoE of the user under challenging handover scenarios while introducing a significant load to the network through file download could yield the true insights

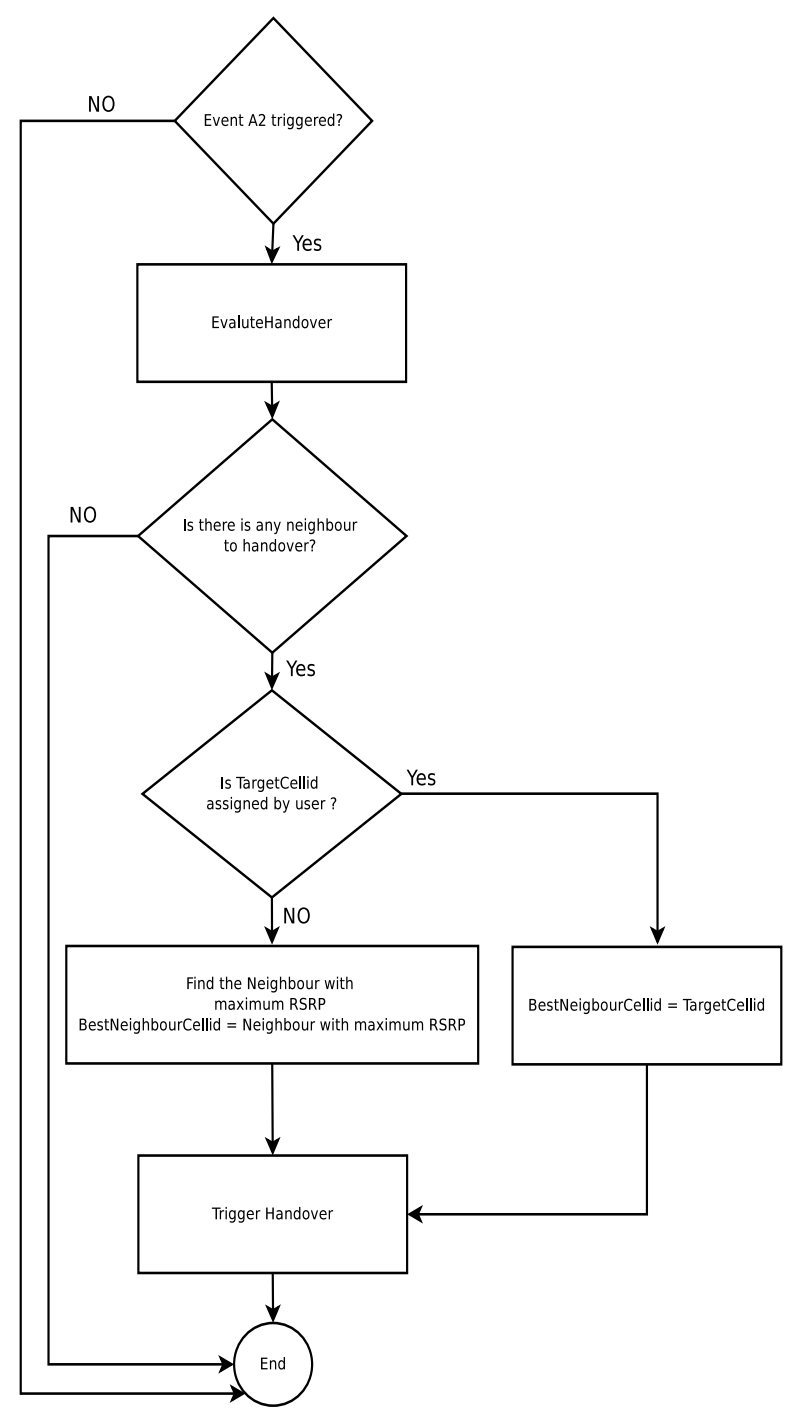

Figure 2: Logic to trigger deterministic handover 
Table 1: Simulation Parameters

\begin{tabular}{|c|c|}
\hline Parameter & Value \\
\hline System bandwidth & $5 \mathrm{MHz}$ \\
\hline Inter-site distance & $500 \mathrm{~m}$ \\
\hline Adaptive Modulation \& Coding Scheme & MiErrorModel \\
\hline Simulation area & $2000 \times 2000 \mathrm{~m}^{2}$ \\
\hline Number of Maco enbs & 3 \\
\hline enbs Tx Power & $46 \mathrm{dBm}$ \\
\hline Number of UEs in system & 3 \\
\hline Velocity of UE1 & $16.6667 \mathrm{~m} / \mathrm{s}$ \\
\hline Path Loss Model & Cost 231 \\
\hline eNB Antenna Height & $30 \mathrm{~m}$ \\
\hline Obstacle Height & $35 \mathrm{~m}$ \\
\hline File Size & $15 \mathrm{MB}$ \\
\hline
\end{tabular}

of the mobility management capabilities of the network.

\subsection{Mobility Model}

To simulate the mobility pattern, we use the RandomWalk2dMobilityModel of ns-3. The purpose of this section is to give an idea of how to configure the RandomWalk2dMobilityModel to achieve the mobility pattern, in which UE picks a fixed starting point and a random destination point following a straight line. With the current model one can tune the attributes as follows,

- "Mode" = Time

- "Time" = Maximum Simulation Time

- "Direction" = Range of angle in radians

The "Mode" attribute indicates the condition for changing the direction and the speed of the UE. For example, in our simulation, by setting it to "Time" which is the maximum simulation time, the UE will not change its speed and direction throughout a single run of the simulation. Additionally, the "Direction" attribute, initialized to the range of angles will enable the UE to have different directions in every run of the simulation. In our simulation, for each run, UE1 picks a fixed starting point close to eNB1, and a random angle in the range of $[+X,-2 X]$, to move away from the source eNB following the straight line; $X$ is the angle from eNB1 to eNB3. This angle can be calculated by applying simple trigonometry to the coordinates of eNB1 and eNB3 as shown in Table. 2 . The range $[+X,-2 X]$ has been chosen by keeping in mind the future work of this study. Where, we intend to use machine learning techniques to build a smart handover algorithm. Thus, we will need all the possible useful mobility patterns to train our smart handover algorithm. As a result, the handover algorithm will learn when the eNB2 is a better choice to handover.

\subsection{Fractional Frequency Reuse}

We use the Hard Fractional Frequency Reuse algorithm of ns-3. By using this algorithm we can divide the whole frequency band into different frequency sub-bands. For exam-

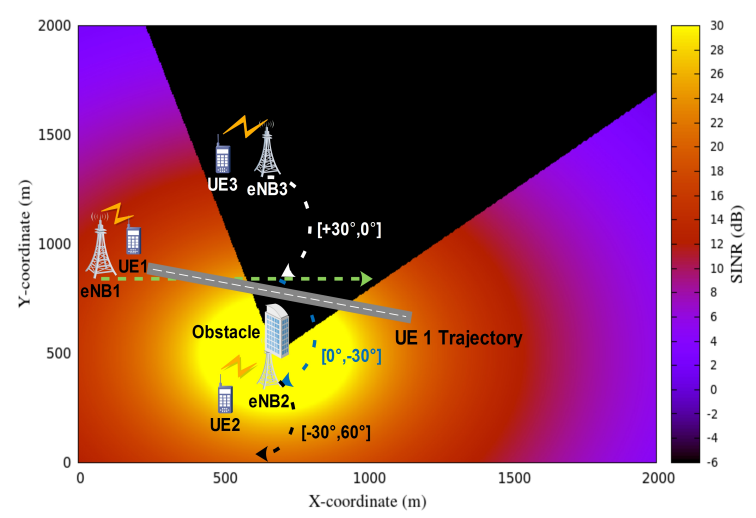

Figure 3: REM for eNB2 with Hard FR algorithm

ple, in this simulation we have 3 eNBs and a system bandwidth of $5 \mathrm{MHz}$, which corresponds to the 25 resource block LTE implementation. This algorithm divides the bandwidth in three sub-bands in such a way, that resource blocks from 1 to 8 are assigned to eNB1, from 8 to 15 to eNB2 and from 16 to 25 to eNB3. To configure the algorithm we have used the automatic configuration as explained in [4]. The frequency reuse in LTE module of ns-3, is only applied to the "Physical Downlink Shared Channel" (PDSCH). Therefore, the key point for this configuration is the selection of CQI feedback generation in the downlink. Currently, in ns-3 there are two approaches to calculate the CQI feedback in the downlink, the Ctrl method and the Mixed method, as explained in [3]. For our simulation we have selected the Mixed method by configuring the UsePdschForCqiGeneration attribute of LteHelper class in following way:

Config::SetDefault ("ns3::LteHelper::UsePdschForCqiGeneration", BooleanValue (true)).

\section{SIMULATION RESULTS}

In Fig. 4 and Fig. 5 we show the simulation results regarding the deterministic handovers to each target eNB. Here we assume that the download time of unfinished downloads are equal to the maximum simulation time. In Fig. 4 we show the download time for two target eNBs, when the angle of direction of the UE movement is in the range of $\left[-30^{\circ}, 0^{\circ}\right]$, as shown in Fig. 3. The download time in case of target eNB2 increases as the UE gets more exposed to the coverage hole and experiences loss of data and huge delays due to long TCP time-outs, which leads to incomplete downloads. For target eNB3 some incomplete downloads are observed due to the high distance from the source eNB. In this case eNB2 is still a better choice due to the reduced

Table 2: Position of Nodes

\begin{tabular}{|c|c|c|c|}
\hline Node & $\mathrm{x}(\mathrm{m})$ & $\mathrm{y}(\mathrm{m})$ & $\mathrm{z}(\mathrm{m})$ \\
\hline eNB1 & 250 & 750 & 30 \\
\hline eNB2 & 683 & 500 & 30 \\
\hline eNB3 & 683 & 1000 & 30 \\
\hline UE1 & 350 & 750 & 1.5 \\
\hline UE2 & 600 & 500 & 1.5 \\
\hline UE3 & 600 & 1000 & 1.5 \\
\hline
\end{tabular}


exposure to the coverage hole. Fig. 5 (a) show the results in terms of average download time for completed downloads and Fig. 5 (b) shows the number of completed/uncompleted downloads, for the complete range of angles with which UE maybe moving. From Fig. 5(a) it can be observed that for the range of $\left[+30^{\circ}, 0^{\circ}\right]$ none of the downloads is completed when handover is done to eNB2, as UE experiences poor channel quality due to its distance from the source cell and the high exposure to the coverage hole. On the other hand, in the range of $\left[-30^{\circ},-60^{\circ}\right]$, none of the downloads get completed when the handover is done to eNB3, as in this case as well, the UE experiences poor channel quality due to its distance from the source cell and due to a small coverage hole between the eNB3 and the UE. The results for the range $\left[0^{\circ},-30^{\circ}\right]$ are very interesting, as state-of-the-art handover algorithms based on the A2 event would provide eNB2 as the strongest candidate for handover, and doing so would cause an increase in average download time and number of incomplete downloads. On the other hand a handover to eNB3 would not only decrease the average download time by $19.5 \%$ but would also decrease the number of incomplete downloads from $46.15 \%$ to $15.3 \%$.

\section{CONCLUSION \& FUTURE WORK}

In this paper, we have presented our approach to the simulation of LTE mobility management scenarios characterized by coverage holes by using LENA LTE module of ns-3. We have described the new features that we added to the ns-3 simulator in order to make this possible, and discussed the details of the configuration of our simulation scenario. Our simulation results have shown that the considered approach is effective for the evaluation of some of the challenging conditions faced by mobility management solutions, when operating in heterogeneous network deployments. We hope that our contribution will help researchers and engineers in using ns-3 to design advanced mobility management solutions that address the poor performance of state-of-the-art handover algorithms. Future works will focus on designing smart handover algorithms based on machine learning techniques that can predict and select the appropriate target cell in order to guarantee the best quality of experience for the user.

\section{ACKNOWLEDGEMENTS}

This work was made possible by NPRP grant No. 5-1047-2437 from the Qatar National Research Fund (a member of

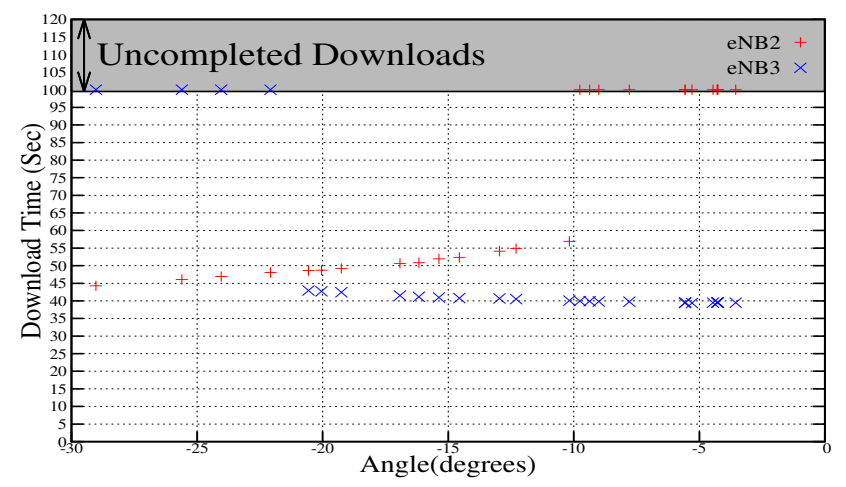

Figure 4: Download time Vs angle

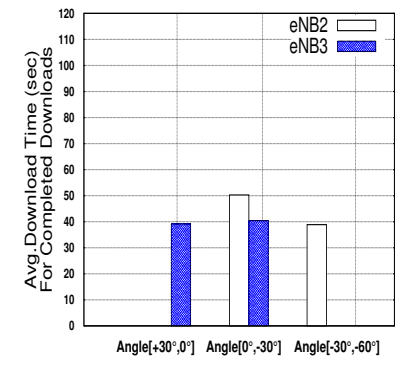

(a)

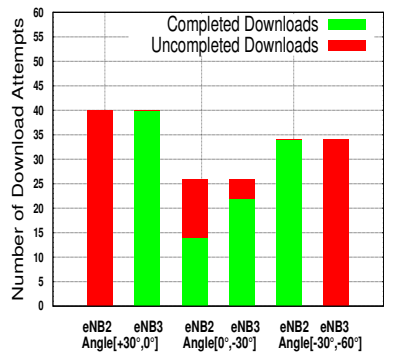

(b)
Figure 5: Average download time for completed downloads (a), Number of completed and uncompleted download attempts(b) vs range of angles.

The Qatar Foundation). The work of Z. Ali is also supported by grant TEC2014-60491-R.

\section{REFERENCES}

[1] Buildings Module Design Documentation. http://www.nsnam.org/docs/release/3.22/models/html /buildings-design.html.

[2] LTE-EPC Network Simulator (LENA). http://networks.cttc.es/mobile-networks/softwaretools/lena/.

[3] LTE Module Design Documentation. http://www.nsnam.org/docs/release/3.22/models/html /lte-user.html.

[4] LTE Module User Documentation. http://www.nsnam.org/docs/release/3.22/models/html /lte-user.html.

[5] 3GPP TS 36.331. Radio Resource Control (RRC); Protocol specification. version 10.12.0, Release 10.

[6] N. Baldo, L. Giupponi, and J. Mangues. Big Data Empowered Self Organized Networks. In Proceedings of the 20th European Wireless Conference, pages 1-8, May 2014.

[7] C. Ericson. Real-Time Collision Detection. Morgan Kaufmann, San Francisco,CA, 2005.

[8] T. Komine, T. Yamamoto, and S. Konishi. A Proposal of Cell Selection Algorithm for LTE Handover Optimization. In Proceedings of the Computers and Communications (ISCC), IEEE Symposium on, pages 37-42, July 2012.

[9] S. S. Mwanje and A. Mitschele-Thiel. Distributed cooperative Q-learning for mobility-sensitive handover optimization in LTE SON. In Proceedings of the Computers and Communication (ISCC), IEEE Symposium on, pages 1-6, June 2014. 